\title{
Commentaires sur le dépouillement des événements acoustiques isolés
}

\author{
M. MAURIN
}

INRETS-LEN, Institut de Recherche sur les Transports et leur Sécurité, Laboratoire Energie Nuisance, Case 24, 69675 Bron cedex, France

\begin{abstract}
We consider isolated noise events, due for instance to isolated vehicles passing in front a sonometer, with an increasing step and a decreasing one. For a time varying level $L(t)=$ $L \max +10 \log b(t)$, (b an attenuation fonction model, $0<b \leq 1$ ), and the noise emergence $a \mathrm{~dB}$ we consider noise equivalent level Leq $=\mathrm{Lmax}-\mathrm{I}(\mathrm{a})$ corresponding to all the delay for which $\mathrm{L}(\mathrm{t})$ $\geq \mathrm{Lmax}-\mathrm{a}$; I(a) is the "intrinsic" relation of the acoustic event, depending of b function. Some properties of $I$ are investigated, it is shown that the influence of $b$ is rather weak for different $b$ models, with no more than $1.6 \mathrm{~dB}$ for an emergence $\mathrm{a}=15 \mathrm{~dB}$.
\end{abstract}

\section{LE MODELE D'UN BRUIT ISOLE.}

Nous considérons la variation temporelle d'un niveau de bruit à propos d'un évènement acoustique isolé qui émerge au dessus du niveau de bruit ambiant, sa signature acoustique est de la forme $L(t)=L \max +$ $10 \log b(t)$ en $\mathrm{dB}, \mathrm{Lmax}$ est un paramètre qui indique le "niveau maximal instantané" et $b(t)$ une application continue positive croissante jusqu'à 1 pour les instants $t$ négatifs - la période de montée du bruit, et décroissante pour $\mathrm{t}$ positif - la période de descente du bruit. Cela peut ainsi représenter le passage d'un véhicule isolé devant un microphone à l'origine du temps t. Bien entendu un niveau instantané n'a pas de sens [1], mais avec une formulation "régulière" (notamment la continuité de la signature) il est possible de parler du paramètre $\mathrm{L} \max =\mathrm{L}(0)$ dans un tel modèle, un paramètre qui a une certaine importance en acous-

tique routière [2].

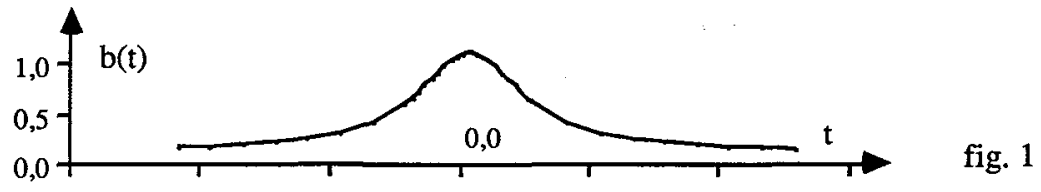

Nous prenons pour simplifier des applications b et $\mathrm{L}$ paires, mais il est immédiat de généraliser aux évènements qui présentent des variations différentes à la montée et à la descente [3], par exemple pour tenir compte de la directivité de la source [2]. En terme de puissance on peut le mettre sous la forme $10^{\mathrm{L} / 10}=$ $10^{\mathrm{Lmax} / 10} \mathrm{~b}(\mathrm{t})$, et sur l'intervalle [- $\left.\mathrm{t} \mathrm{t}\right]$, $\mathrm{t}$ positif, le niveau de bruit équivalent Leq est donné par la relation de définition $10^{\mathrm{Leq} / 10}=1 / 2 \mathrm{t} 10^{\mathrm{Lmax} / 10} \int_{-t}^{\mathrm{t}} \mathrm{b}(\mathrm{u}) \mathrm{du}=10^{\mathrm{Lmax} / 10} \mathrm{~B}(\mathrm{t}) / \mathrm{t}$ en posant $\mathrm{B}(\mathrm{t})=\int_{0}^{\mathrm{t}} \mathrm{b}(\mathrm{u}) \mathrm{du} . \mathrm{Par}$ conséquent on aboutit aux variations temporelles $L(t)=L \max +10 \log b(t)$ et $\left.\operatorname{Leq}_{[-t} t\right]=L \max +$ $10 \log B(t) / t$ en fonction du temps. 
Lemme 1 : $S i b(t)$ est positive décroissante sur $[0+\infty[$ alors pour tout $t$ postif l'application $B(t) / t$ est décroissante en $t$ et vérifie la relation $1 \geq B(t) / t \geq b(t) \geq 0$.

En effet puisque $b$ est décroissante pour $\tau$ positif on a nécessairement $t b(0)=t \geq B(t) \geq t b(t)$ (premier théorème de la moyenne) ce qui entraîne l'inégalité ;

par ailleurs $\left(\frac{B}{t}\right)^{\prime}=\frac{1}{t^{2}}(t b-B)$ est de signe négatif ou nul, et donc $B / t$ décroît en fonction de $t$.

On peut également étudier le comportement de $\mathrm{B} / \mathrm{t}$ au voisinage de l'origine et de plus l'infini.

i) au voisinage de l'origine : avec l'hypothèse de continuité de $b$ à l'origine, $b$ et $B / t$ tendent toutes deux vers 1 quand $t$ tend vers zéro, (que b soit paire ou non d'ailleurs);

en ce qui concerne des régularités d'ordres supérieurs :

* si b deux fois dérivable à l'origine, ce qui n'est possible par parité qu'avec une dérivée $b^{\prime}(0)$ nulle, cela entraîne que $B / t$ est dérivable à l'origine avec une dérivée nulle également ; $b$ possède un rayon de courbure $R$ à l'origine, son développement limité est de la fome $1-t^{2} / 2 R+O\left(t^{3}\right)$, et celui de $B / t$ de la forme $1-\mathrm{t}^{2} / 6 \mathrm{R}+\mathrm{O}\left(\mathrm{t}^{3}\right)$ avec un rayon de courbure à l'origine 3 fois plus grand que celui de $\mathrm{b}$;

* nous supposons que la variation de b présente un point singulier "anguleux" à l'origine avec une dérivée à droite et une à gauche, et un développement limité de la forme $b=1-c \mid t I+O\left(t^{2}\right)$, auquel cas le développement limité de $\mathrm{B} / \mathrm{t}$ est de la forme $1-\mathrm{c} / 2 \mathrm{t} \mid+\mathrm{O}\left(\mathrm{t}^{2}\right)$, et présente un point singulier de même nature ; ii) au voisinage de plus l'infini : puisque b est décroissante $B / t$ l'est également, les applications b et $B / t$ tendent vers la même limite positive ou nulle lorsque $t$ tend vers plus l'infini ; dans la suite nous supposons que l'énergie du signal est finie - b intégrable - et donc que la limite commune est égale à zéro.

\section{DEFINITION DE LA COURBE INTRINSEQUE.}

2.1 Sur [- $t \mathrm{t}$ ] le niveau L subit l'atténuation maximale, ou émergence, $10 \log b(t)$ tandis que le Leq $[-t]$ correspondant ne varie que de $10 \log \mathrm{B} / \mathrm{t}$. Par définition la "courbe intrinsèque" de l'évènement acoustique isolé est la variation de $I=-10 \log B / t$ du niveau équivalent en fonction de l'émergence $10 \log b(t)$. Pour plus de commodité nous posons $-10 \log b=a, a>0$, d'où $t(a)=b^{-1}\left(10^{-a / 10}\right)$, d'où l'expression de la courbe intrinsèque $I(a)=-10 \log \frac{B(t(a))}{t(a)}$. La coure intrinsèque possède une signification physique et pratique tant que le niveau Lmax - a de la signature de l'évènement est supérieur au bruit de fond

Lemme 2 : La courbe intrinsèque est positive croissante définie pour tout a positif.

En effet I est positive puisque $B / t$ est inférieur à 1, définie pour tout t positif et donc a positif ; par ailleurs $\mathrm{I}(\mathrm{a})^{\prime}=-\frac{10}{\log 10} \frac{(\mathrm{B} / \mathrm{t})_{\mathrm{t}}{ }^{\prime}}{\mathrm{B} / \mathrm{t}} \frac{\mathrm{dt}}{\mathrm{da}}$, avec $\mathrm{B} / \mathrm{t}$ une application décroissante et $\mathrm{t}(\mathrm{a})$ une application croissante, il en résulte que le signe de I(a)' est positif.

La courbe intrinsèque peut aussi être étudiée quand l'argument a tend vers zéro ou vers plus l'infini. i) au voisinage de $+\infty$, lorsque a tend vers $+\infty, 10^{-a / 10}=b$ tend vers zéro, $t$ tend vers $+\infty, b$ et $B / t$ tendent vers zéro et $I$ tend donc vers $+\infty$. Si en outre nous supposons que l'évènement possède une énergie finie, c'est à dire que l'intégrale $\int_{0}^{\infty} \mathrm{b}(\mathrm{u})$ du est une constante finie $\mathrm{K}$, alors l'atténuation I tend vers $+\infty$ comme $10 \log \mathrm{t}(\mathrm{a})-10 \log \mathrm{K}$, par exemple :

* b décroît comme $\mathrm{e}^{-t}$, alors $\mathrm{t}$ croît comme $\frac{\log 10}{10} \mathrm{a}$, et $\mathrm{I}$ comme $10 \log \mathrm{a}$;

* b décroît comme $\mathrm{t}^{-\gamma} \gamma>1$, alors $\mathrm{t}$ croît comme $10^{\mathrm{a} / 10 \gamma}$, et I comme $\mathrm{a} / \gamma$; 
ii) au voisinage de l'origine.

Lorsque a tend vers $0,10^{-a / 10}=b$ tend vers $1, t$ tend vers $0, b$ et $B / t$ tendent vers 1 et $I$ tend donc vers 0 . * lorsque le développement limité de b est de la forme $1-t^{2} / 2 R+O\left(t^{3}\right)$, celui de $B / t$ de la forme $1-t^{2} / 6 R$ $+O\left(t^{3}\right)$, a croît comme $\frac{10}{\log 10} \frac{t^{2}}{2 R}$ et I comme $a / 3$; en particulier la valeur de $R$ n'intervient pas ;

* lorsque le développement limité de b est de la forme $1-\mathrm{ct}+\mathrm{O}\left(\mathrm{t}^{2}\right), t$ positif, celui de $\mathrm{B} / \mathrm{t}$ de la forme $1-c / 2 t+O\left(t^{2}\right)$, a crôt comme $\frac{10}{\log 10}$ ct et $I$ comme $a / 2$; la valeur de $c$ n'intervient pas.

On observe que c'est essentiellement la qualité de b à l'origine (courbure ou non) qui intervient dans les premières variations de I, et non pas les valeurs de la courbure $\mathrm{R}$ ou de la tangente à droite $c$.

2.2 Exemples, (figure 2).

1) l'atténuation classique en champ libre $b=\frac{1}{1+t^{2}}$ auquel cas $B(t)=$ Arctg $t$. Dans ce cas les calculs sont explicites et donnent $\frac{1}{1+\mathrm{t}^{2}}=10^{-\mathrm{a} / 10}, \mathrm{t}=\sqrt{10^{\mathrm{a} / 10}-1}$ puis $\mathrm{I}(\mathrm{a})=-10 \log \left\{\frac{\operatorname{Arctg} \sqrt{10^{\mathrm{a} / 10}-1}}{\sqrt{10^{\mathrm{a} / 10}-1}}\right\}$;

2) $b=c^{-|t|}=e^{-|t| \log c}, B(t)=\left(1-c^{-t}\right) / \log c ;$ les calculs explicites donnent $t=\frac{a \log 10}{10 \log c}$ puis $I(a)=$ $-10 \log \left\{\frac{10}{a \log 10}\left(1-10^{-a / 10}\right)\right\}$ qui ne dépend pas de $c ; b$ n'a pas de courbure à l'origine ;

Les exemples suivants sont menés avec des intégrations numériques [3].

3) l'atténuation de champ libre complétée par une exponentielle décroissante $b=\frac{1}{1+t^{2}} e^{\mu} e^{-\mu \sqrt{ }\left(1+t^{2}\right)}$ avec un coefficient $\mu$ d'atténuation linéaire ;

4) l'atténuation de Bullen et Fricke est utilisée dans les milieux en périphérie urbaine dont les façades sont disposées de manière irrégulière [4]. Elle est de la forme $b(t)=\frac{1}{K_{1}(1)} \frac{K_{1}(u)}{u}$ avec $K_{1}$ la fonction de Bessel modifiée de deuxième espèce du premier ordre [5] et l'argument $u(t)=\sqrt{1+t^{2}}$. Cette atténuation est intégrable, $\int_{0}^{\infty} b\{u(t)\} d t=\int_{1}^{\infty} b(u) \frac{u d u}{\sqrt{u^{2}-1}}=\int_{1}^{\infty} \frac{K_{1}(u)}{K_{1}(1)} \frac{d u}{\sqrt{u^{2}-1}}$ est définie puisque $K_{1}(x)$ est $\mathrm{O}\left(\mathrm{e}^{-\mathrm{x}} \mathrm{x}^{-1 / 2}\right)$ au voisinage de $+\infty[5]$; on vérifie qu'elle a une courbure à l'origine ;

5) l'atténuation de Kuttruff utilisée pour les rues de centre ville avec réflexions sur des façades rectilignes parallèles [6,7]. Elle est de la forme $b(t)=\frac{1}{f(1)} \frac{f(u)}{u}$ avec $u=\sqrt{1+t^{2}}$ et l'application $f(u)=\int_{0}^{\infty} \frac{e^{-u x}}{1+x^{2}} d x$ [5]. Cette atténuation est intégrable, $\int_{0}^{\infty} b\{\mathrm{u}(\mathrm{t})\} \mathrm{dt}=\int_{1}^{\infty} \mathrm{b}(\mathrm{u}) \frac{\mathrm{u} \mathrm{du}}{\sqrt{\mathrm{u}^{2}-1}}=\int_{1}^{\infty} \frac{\mathrm{f}(\mathrm{u})}{\mathrm{f}(1)} \frac{\mathrm{du}}{\sqrt{\mathrm{u}^{2}-1}}$ est définie puisque $\mathrm{f}(\mathrm{x})$ est $\mathrm{O}\left(\mathrm{x}^{-2}\right)$ au voisinage de $+\infty[5]$, et elle a une courbure à l'origine.

\subsection{Premiers enseignements.}

L'observation des variations graphiques, l'étude de leurs variations à l'origine, et diverses propriétés annexes, [3] : invariance en fonction du changement d'échelle temporelle $t$ en $\eta t$ (la vitesse du véhicule par exemple), effet "médian" de la non symétrie, montrent que les courbes intrinsèques avec des b théoriques comme ci-dessus ne dépendent pas très fortement du modèle. C'est la présence ou l'absence de courbure à l'origine pour $\mathrm{b}$ qui a le plus d'effet pour les faibles émergences. Pour une émergence inférieure à $10 \mathrm{~dB}$ les courbes figurées ne different pas de plus de $\Delta \mathrm{I}=1 \mathrm{~dB}$, et de $1,6 \mathrm{~dB}$ pour une émergence de $15 \mathrm{~dB}$. 
On remarque ainsi que la connaissance précise des courbes de montée et de descente de la signature $10 \log b$ influencent assez peu la valeur du niveau équivalent Lmax - I(a) rapporté à l'émergence a correspondant à la mesure du niveau équivalent, avec des $\Delta \mathrm{I}(\mathrm{a})$ inférieurs à $2 \mathrm{~dB}$ pour $\mathrm{a} \leq 15 \mathrm{~dB}$.

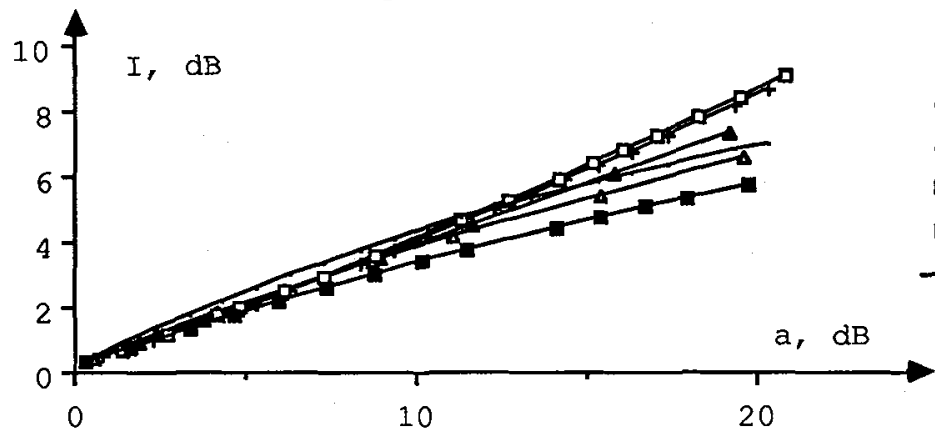

+ champ libre

- ch.1. $+\mu=0,1$

$\triangle \quad$ ch.1. $+\mu=0,5$

auttruff

- Bullen Fricke

$-\exp (-\mathrm{t})$

0

fig. 2

En parallèle nous avons examiné des enregistrements de quelques évènements isolés (ici des montées et descentes des signatures de tramways $n^{\circ} 15,24$ ), et calculé les courbes intrinsèques correspondantes. Elles ont une allure plus heurtées - ce qui est naturel compte tenu des oscillations qui entachent en pratiqure les signatures temporelles - mais elles sont aussi plus faibles et dispersées que celles du faisceau précédent, et assez proches pour certaines de l'atténuation $b=\exp \left(-t^{2}\right)$ qui n'a guère de fondement acoustique, fig. 3 ; cette dernière augmente les écarts $\Delta \mathrm{I}(10)$ et $\Delta \mathrm{I}(15)$ de 0,5 et $1,1 \mathrm{~dB}$ environ.

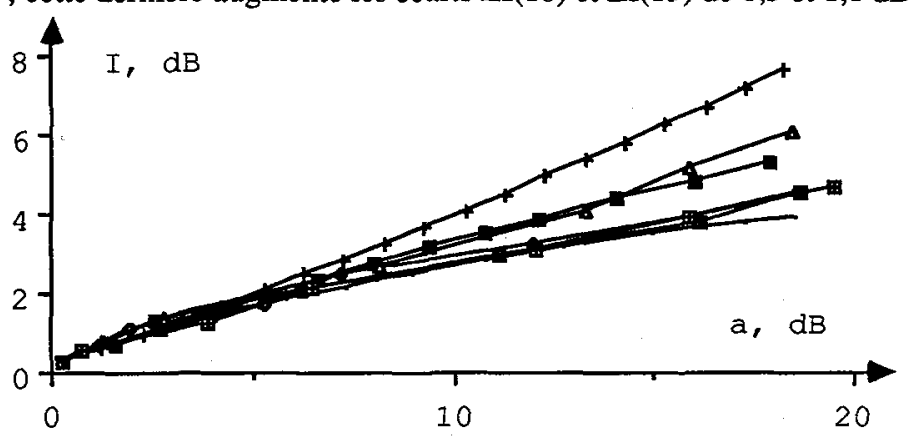

$+\quad$ champ libre

- Bullen Fricke

- $\exp (-\mathrm{t} 2)$

- 24 descente

A 24 montée

- 15 descente

15 montée

fig. 3

On observe des courbes intrinsèques expérimentales qui ne sont pas tout à fait en accord avec les courbes qui résultent des modèles "b" de la littérature. L'étude d'autres propriétés analytiques des courbes intrinsèques attachées à un évènement acoustique isolé est en cours [3], le concept mérite aussi un complément d'examen pour s'assurer qu'il présente des applications pratiques, et notamment l'étude d'une relative insensibilité en fonction de la signature.

\section{Références}

[1] Maurin M., 1992, Qu'est ce qu'une pression acoustique instantanée, ..., Journal d'Acoustique, vol 5 n 6, 639-648.

[2] Hamet J.F., 1993, Niveau de bruit d'un véhicule au passage ..., Journée spécialisée INRETS-MMA, Lyon, 25 nov 93.

[3] Maurin M., 1993, L'analyse des niveaux de bruit isolés, note de calcul, INRETS-LEN.

[4] Bullen R., Fricke F.R., 1979, Traffic noise in urban areas, Australian road research, vol 9 n $^{\circ} 4,11-15$.

[5] Abramowitz M., Stegun, I.A., 1970, Handbook of mathematical functions, Dover.

[6] Kuttruff H., 1975, Zur berechnung von pegelmittelwerten und schwankungsgrössen ..., Acustica, vol 32, $n^{\circ} 2,57-69$.

[7] Otha M., Yamaguchi S., Ikuta A., 1980, Statistical estimation of road traffic noise in an arbitrary sound propagation environment by use of Stratonovich's theory ..., JSV, vol $69 n^{\circ} 2,275-284$. 